Research Paper

\title{
Systematic Review and Meta-Analysis on the Association between Polymorphisms in Genes of IL-12 Signaling Pathway and Hepatocellular Carcinoma Risk
}

\author{
Yao Xiao1\#, Guodong Liu²\#, Liansheng Gong1 ${ }^{\bowtie}$ \\ 1. Department of Hepatobiliary and Pancreatic Surgery, Xiangya Hospital, Central South University, Changsha, Hunan \\ 2. Department of Pancreatic Biliary Surgery, Xiangya Hospital, Central South University, Changsha, Hunan \\ \#These authors contributed equally to the work. \\ $\triangle$ Corresponding author: Liansheng Gong (Department of Hepatobiliary and Pancreatic Surgery, Xiangya Hospital, Central South University, Xiangya Road 87, \\ Changsha, Hunan 410008, People's Republic of China). Tel: +86 7318975 3060. Email: gongliansheng8280@163.com \\ (C) Ivyspring International Publisher. This is an open access article distributed under the terms of the Creative Commons Attribution (CC BY-NC) license \\ (https://creativecommons.org/licenses/by-nc/4.0/). See http://ivyspring.com/terms for full terms and conditions.
}

Received: 2018.04.01; Accepted: 2018.08.02; Published: 2018.09.08

\begin{abstract}
We performed an updated meta-analysis and systematic review to explore the associations between polymorphisms in genes of IL-12 signaling pathway and hepatocellular carcinoma (HCC) risk. Diverse databases were retrieved to identify entire available studies, and odds ratios (ORs) correspondence with $95 \%$ confidence intervals (Cls) were performed to assess their associations. Finally, 6 polymorphisms in five genes of the IL-12 signaling pathway were extracted from 39 case-control studies, 26 publications. We identified that STAT4-rs7574865 polymorphism was significantly associated with an increased risk of HCC in allelic contrast, dominant, homozygote and recessive models. However, we failed to uncover any significant association between other polymorphisms in genes of IL-12 signaling pathway and HCC risk, including ILI8-rs1946518 and -rs187238, IFN- $\gamma$-rs2430561, ILI 2A-rs568408, ILI2B-rs3212227 and STAT4-rs7574865. When the subgroup analysis was conducted based on Hardy-Weinberg Equilibrium (HWE) status, we identified that IFN- $\gamma$-rs 2430561 polymorphism was significantly associated with an increased risk of $\mathrm{HCC}$ in homozygote and recessive models of these studies whose control groups were conformed to HWE. To sum up, our study suggests that STAT4-rs7574865 is a risk factor for HCC. Further well-designed large sample size studies are warranted to shed new light on these findings.
\end{abstract}

Key words: IL-12 signaling pathway; polymorphism; Hepatocellular Carcinoma; risk

\section{Introduction}

Primary liver cancer is the sixth most frequent cancer around the world and the second ordinary cause of cancer-related death. Of them, approximately 70 to $85 \%$ of primary liver cancer cases are hepatocellular carcinoma (HCC) $[1,2]$. Due to the high infection of hepatitis $\mathrm{B}$ virus (HBV), the high prevalence rate of HCC was observed in East Asia, Southeast Asia and sub-Saharan Africa [3-5]. In 2015, about 500,000 individuals were newly diagnosed, and lead to 420,000 death in China [6-9].

Cytokines are a family of proteins, which are familiarly concerned with both innate and adaptive immune responses to fight against infections. With the background of chronic hepatic inflammation, cytokines comprehensively participate in tumorigenesis process, including IL-6, IL-12, IL-18 and etc. [10-12]As a key immunoregulatory cytokine, IL-12 is consisted of two subunits, IL-12-p35 and IL-12-p40, which are translated from IL-12A gene and IL-12B gene, and were link with each other through covalent bond [13, 14]. IL-12 is an early pro-inflammatory cytokine, mainly secreted by antigen-presenting cells to amplify inflammatory signals. When IL-12 binds to IL-12R complex, the JAK 
kinase (Tyk-2 and Jak-2) will be activated, thus contributes to the phosphorylation of IL-12R. Study also demonstrated that tyrosine phosphorylation of STAT4 protein, another pivotal molecular of IL-12 signaling pathway, which could regulate gene transcription through DNA homodimerization or translocation in nucleus [15]. IL-12 also induces the expression of IFN- $\gamma$ in $\mathrm{T}$ and NK cells through activating JAK/STAT4 pathway and plays a fundamental role in the differentiation of naive T cells to Th1 cells [16, 17]. As a synergistic manner, IL-18 could together with IL-12 stimulates IFN- $\gamma$ production by Th1 and NK cells, in addition, IL-12 could also up-regulate IL-18R expression promoting the secreting of IFN- $\gamma$ [18].

As for IL-18, which is a cytokine initially known as an inducer of IFN- $\gamma$, plays important roles during both Th1 and Th2 responses [19]. These studies demonstrated that genes of IL-12 signaling pathway could functionally work together, contributing to the anti-infection process, and dysregulation of one or more genes in this pathway potentially can influence the whole pathway and thus result in tumorigenesis process. In addition, more evidence has been pointed out that IL-12 signaling pathway plays a pivotal role during anti-HBV-infection, and might contribute to the HCC pathogenesis [20, 21].

Till now, plenty of studies have examined the associations between polymorphisms in genes of IL-12 signaling pathway and HCC risk, however, these results were controversial and inconsistent. Such as, for IL12B-rs3212227 polymorphism, in Yang et al.'s [22] work, they suggested that this genetic polymorphism may have an independent effect HCC risk in a Chinese population, on the contrary, another study showed that it has no statistically difference between HCC cases and cancer-free chronic HCV patient groups[23]. As for STAT4, Chanthra et al. [24] found out that STAT4-rs7574865 polymorphism was related to an increased risk of HCC progression, a results consistent with Clark et al.'s work [25]. However, in another study conducted by Chen et al.[26], they failed to validate the function of rs7574865 polymorphism in STAT4 on the risk of HCC. Due to the heterogeneity within cancer subtypes, the diverse ethnicities of patient cohorts and the small sample sizes, the studies concerned about polymorphisms in genes of IL-12 signaling pathway and HCC risk were not consistent. To overcome these limitations, we exhaustively collected all available genetic polymorphisms in genes of IL-12 signaling pathway and their relevant eligible studies about HCC risk, and performed an updated meta-analysis to comprehensively demonstrate the associations between genetic variations of genes in IL-12 signaling pathway and HCC risk.

\section{Materials and Methods}

\section{Literature filtrating and distinguishing of relevant studies}

In order to identify all available studies regarding the relationships between genetic polymorphisms in genes of IL-12 signaling pathway and HCC risk, comprehensively literature search was conducted on diverse online databases, including PubMed, Embase, Science Direct and Google Scholar published up to May 30, 2018 by applying below MeSH terms: ('genes' OR 'abbreviations of genes') AND ('cancer' OR 'adenocarcinoma' OR 'tumor' OR 'carcinoma' OR 'neoplasms') AND ('variant' OR 'mutation' OR 'polymorphism' OR 'SNP' OR 'genotype'). Language of eligible studies was restricted to English and Chinese. All of the retrieved articles were reviewed by reading the title and abstract. In addition, full texts of these possibly relevant studies were further read for suitability in current work. Furthermore, in order to identify more eligible studies, the references of each enrolled study were also searched manually.

\section{The Criteria of Inclusion and Exclusion}

Publications inclusion criteria were demonstrated as: (1) patients were diagnosed by histopathology testing, and control group should be cancer-free, age-matched and sex-matched; (2) case-control studies which focus on the associations between polymorphisms in genes of IL-12 pathway and HCC risk; (3) enrolled articles should have sufficient genotype data, in order to calculate odds ratios (ORs) and 95\% confidence intervals (CIs). On the contrast, publications should be excluded when they were: (1) Reviews or conference papers; (2) only case study; or (3) have no sufficient data.

\section{Extracting of Data and Assessing of Article Quality}

Data extraction and quality evaluation of each enrolled publications were conducted by Yao Xiao and Guodong Liu, independently. All the disagreements should be solved after discussion. Furthermore, the following information will be extracted from each publication, including name of the first author, publication year, ethnicity, allele and genotype distribution and Hardy-Weinberg equilibrium (HWE).

\section{Meta-Analysis}

The associations between polymorphisms in gene of IL-12 signaling pathway and HCC risk was assessed by ORs and $95 \% \mathrm{CI}$. And the significance of 
pooled ORs was determined by Z-test. Bonferroni correction was applied to adjust the $P$-value of Z-test, and $P$-adjust less than $1.67^{*} 10^{-3}[0.05 /$ (five genetic models * six polymorphisms)] was considered as statistical significant[27]. Five genetic models were used to calculate their associations, including allele (M vs. W), homozygous (MM vs. WW), heterozygous (MW vs. WW), dominant (MW + MM vs. WW), and recessive models (MM vs. WW + WW) ( $\mathrm{W}$ refers to wild allele and $M$ refers to mutated allele). After that, stratified analyses were also conducted by different cancer type, ethnicity or source of control. Heterogeneity assumption was checked by $\mathrm{I}^{2}$ test and $Q$ statistic test. When $\mathrm{I}^{2} \leq 50 \%$ and $P \geq 0.1$, the heterogeneity could be ignored, then, the fixed-effect model will be applied; Otherwise, the random-effect model will be selected [28]. Moreover, publication bias was appraised with the help of Egger's regression test and Begg's funnel plot, and the stability of results

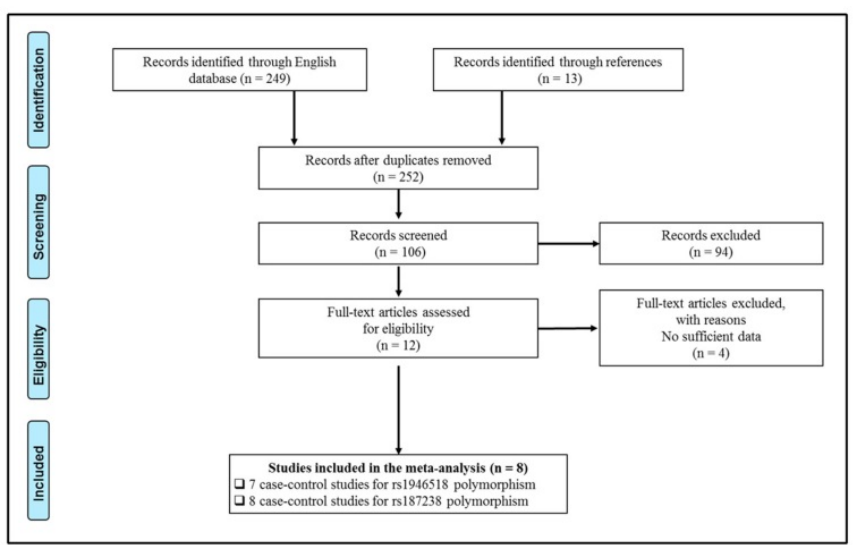

IL18

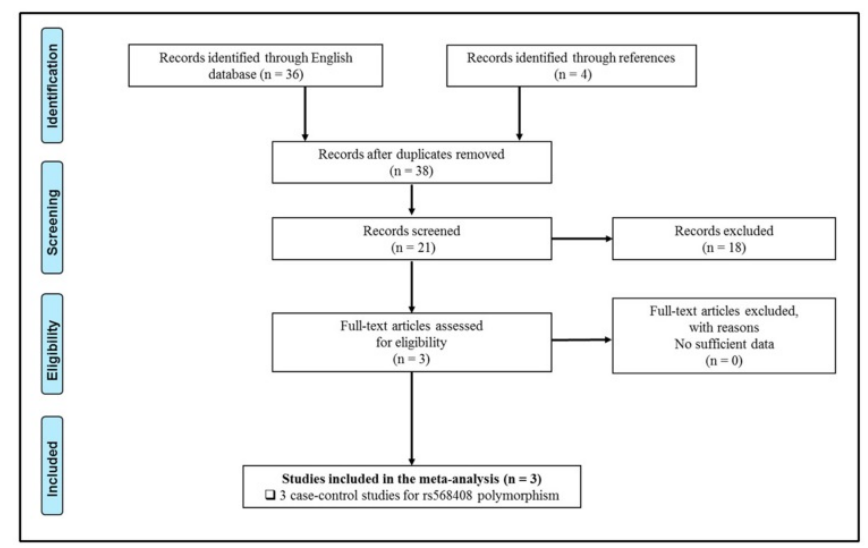

IL12B was confirmed by sensitivity analysis [29]. All statistical analyses were conducted by the Stata software (version 12.0; Stata Corporation, College Station, TX).

\section{Results}

\section{Study identification and characteristics of enrolled publications}

347 publications were identified after initial screening. After scoring out duplicates by reading the titles and abstracts, 310 publications were removed. Then, 37 full-text publications were assessed for eligibility. Among them, 11 publications were further excluded because relevant studies for one polymorphism were less than three. Finally, 26 publications comprising 39 case-control studies were enrolled for current meta-analysis, and the study selection process was presented in Figure 1.

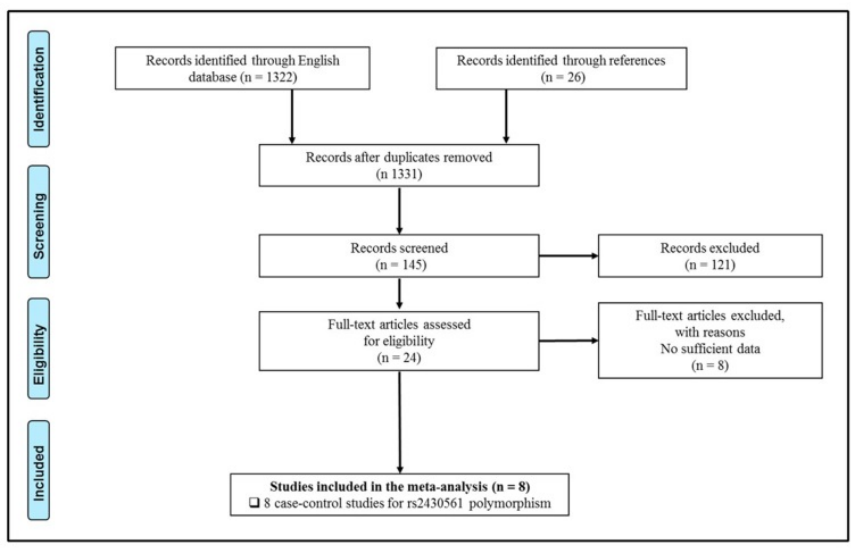

IFN- $\gamma$

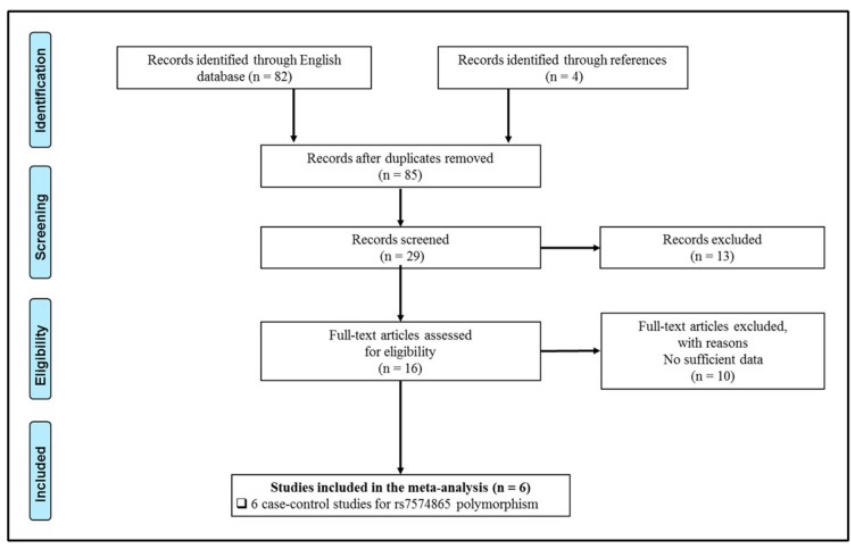

STAT4 


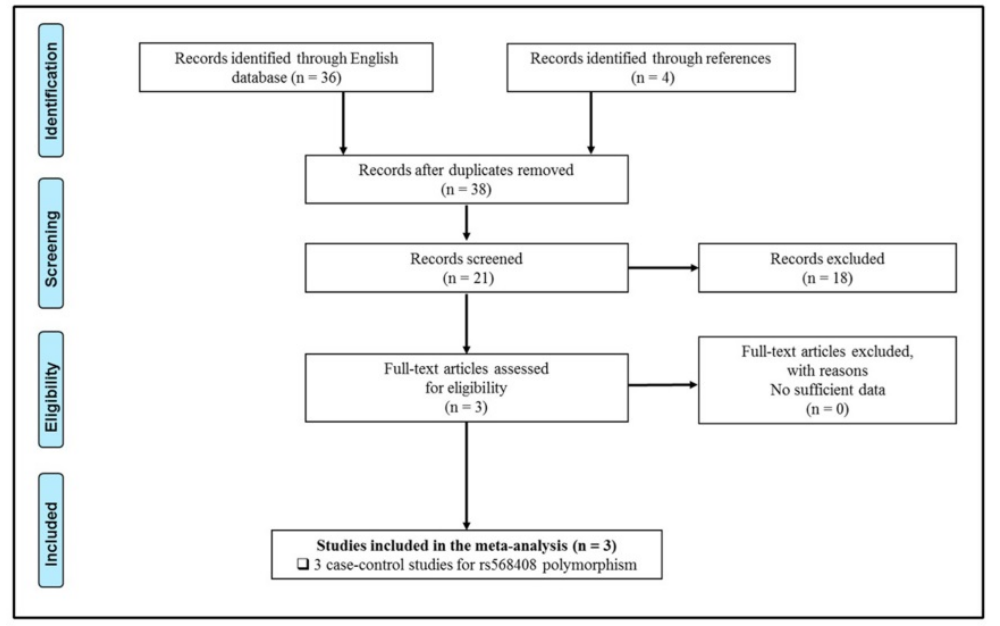

IL12A

Figure 1. Study selection process for each gene enrolled.

The general demographical characteristics of all eligible publications were summarized in Table 1, including IL18-rs1946518/ rs187238 [30-37], IFN- $\gamma^{-}$ rs2430561 [35, 38-44], IL12A-rs568408 [45-47], IL12B-rs3212227 [42, 43, 45-49] and STAT4-rs7574865 $[25,26,50-53]$. In addition, the quality of each enrolled study was assessed by Newcastle-Ottawa Scale (NOS), and the outcomes were presented in (Table S1).

\section{Meta-analysis}

The detail results of current meta-analysis were shown in Table 2 . The overall results suggested that STAT4-rs7574865 polymorphism conferred a statistically increased risk of HCC in allelic (M vs. W: $\left.\mathrm{OR}=1.270,95 \% \mathrm{CI}=1.166-1.384, P_{\mathrm{A}}=1.760 \times 10^{-8}\right)$, homozygous $(\mathrm{MM}$ vs. $\mathrm{WW}$ : $\mathrm{OR}=1.651,95 \% \mathrm{CI}$ : 1.352-2.016, $\left.P_{\mathrm{A}}=6.561 \times 10^{-7}\right)$, recessive $(\mathrm{MM}$ vs. $\mathrm{MW}+\mathrm{WW}: \mathrm{OR}=1.330,95 \% \mathrm{CI}=1.168-1.516, P_{\mathrm{A}}=$ $\left.4.680 \times 10^{-7}\right)$ and dominant models $(\mathrm{MW}+\mathrm{MM}$ vs. $\mathrm{WW}: \mathrm{OR}=1.470,95 \% \mathrm{CI}=1.213-1.781, P_{\mathrm{A}}=$ $\left.7.092 \times 10^{-5}\right)$. Moreover, subsection analysis performed on source of control demonstrated that the P-B groups were more susceptible to develop HCC in allelic (M vs. $\mathrm{W}: \mathrm{OR}=1.320,95 \% \mathrm{CI}=1.193-1.461, P_{\mathrm{A}}=$ $\left.7.210 \times 10^{-8}\right)$, homozygous (MM vs. WW: $\mathrm{OR}=1.687$, 95\%CI: 1.326-2.146, $\left.P_{\mathrm{A}}=1.593 \times 10^{-5}\right)$, recessive $(\mathrm{MM}$ vs. $\mathrm{MW}+\mathrm{WW}: \mathrm{OR}=1.417,95 \% \mathrm{CI}=1.242-1.616, P_{\mathrm{A}}=$ $\left.1.961 \times 10^{-7}\right)$ and dominant models $(\mathrm{MW}+\mathrm{MM}$ vs. $\mathrm{WW}: \mathrm{OR}=1.447,95 \% \mathrm{CI}=1.148-1.824, P_{\mathrm{A}}=1.354 \times 10^{-3}$, Figure 2), respectively. However, negative results were identified when subgroup analyses were conducted based on HWE, ethnicity and source of control.

Although overall results failed to uncover any positive association between IL18-rs1946518/ -rs187238, IFN- $\gamma$-rs2430561, IL12A-rs568408 and
IL12B-rs3212227 polymorphisms and HCC risk, similar to STAT4-rs7574865 polymorphism, we identified that IL18-rs187238 polymorphism was related to an increased risk of HCC in H-B groups in allelic (M vs. $\mathrm{W}: \mathrm{OR}=1.604,95 \% \mathrm{CI}=1.223-2.103, P_{\mathrm{A}}=$ $7.535 \times 10^{-4}$ ), heterozygous (MW vs. WW: $\mathrm{OR}=1.665$, 95\%CI: $\left.1.227-2.258, P_{\mathrm{A}}=1.058 \times 10^{-3}\right)$ and dominant models $(\mathrm{MW}+\mathrm{MM}$ vs. $\mathrm{WW}: \mathrm{OR}=1.678,95 \% \mathrm{CI}=$ 1.246-2.260, $\left.P_{\mathrm{A}}=6.950 \times 10^{-4}\right)$. For $I F N-\gamma$-rs 2430561 polymorphism, although overall analysis failed to uncover any positive result, when the subgroup analysis was conducted based on HWE status, we found that for these studies whose control groups conformed to HWE, were significantly associated with an increased risk of HCC in homozygous (MM vs. $\mathrm{WW}: \mathrm{OR}=2.375,95 \% \mathrm{CI}=1.393-4.050, P_{\mathrm{A}}=$ $\left.1.450 \times 10^{-3}\right)$ and recessive models (MM vs. MM+MW: $\left.\mathrm{OR}=2.331,95 \% \mathrm{CI}=1.471-3.691, P_{\mathrm{A}}=2.991 \times 10^{-4}\right)$, respectively.

\section{Sensitivity Analysis and Publication Bias}

Sensitivity analyses were performed to assess the impact of separate case-control study on the data pools (including IL18-rs1946518/rs187238, IFN- $\gamma$ rs2430561, IL12A-rs568408, IL12B-rs3212227 and STAT4-rs7574865 polymorphisms), and the results showed that the pooled ORs and 95\%CIs were not been significantly influenced after removing each case-control study in sequence (Table S2 and Figure S1). Moreover, to evaluate the publication bias, Begg's funnel plot and Egger's regression test were performed for each genetic polymorphism. By observing the shape of Begg's funnel plot, no evidence of publication bias was identified for any polymorphism, which was further verified by Egger's regression test (Table S3 and Figure S2). 
Table 1. Characteristics of eligible enrolled studies.

\begin{tabular}{|c|c|c|c|c|c|c|c|c|c|c|c|c|}
\hline \multirow[t]{2}{*}{ Gene } & \multirow[t]{2}{*}{ Polymorphism } & \multirow[t]{2}{*}{ First author } & \multirow[t]{2}{*}{ Year } & \multirow[t]{2}{*}{ Ethnicity } & \multirow[t]{2}{*}{ Source of Control } & \multirow[t]{2}{*}{ Cancer Type } & \multicolumn{3}{|l|}{ Case } & \multicolumn{3}{|c|}{ Control } \\
\hline & & & & & & & WW & WM & MM & WW & WM & $\mathbf{M M}$ \\
\hline \multirow[t]{15}{*}{ IL18 } & rs1946518 & Teixeira et al.[35] & 2013 & mixed & P-B & $\mathrm{HCC}$ & 38 & 56 & 18 & 85 & 105 & 12 \\
\hline & rs1946518 & Lau et al..$^{27}$ & 2016 & Asian & H-B & $\mathrm{HCC}$ & 88 & 167 & 87 & 148 & 276 & 135 \\
\hline & rs1946518 & Bao et al. 25 & 2015 & Asian & P-B & $\mathrm{HCC}$ & 37 & 73 & 43 & 41 & 76 & 48 \\
\hline & rs1946518 & Migita et al..$^{29}$ & 2009 & Asian & P-B & $\mathrm{HCC}$ & 13 & 26 & 8 & 20 & 30 & 13 \\
\hline & rs1946518 & Chen et al. ${ }^{26}$ & 2012 & Asian & P-B & $\mathrm{HCC}$ & 47 & 126 & 55 & 83 & 156 & 61 \\
\hline & rs1946518 & Karra et al..$^{28}$ & 2015 & African & P-B & $\mathrm{HCC}$ & 70 & 152 & 49 & 102 & 144 & 34 \\
\hline & rs1946518 & Zhang et al. ${ }^{32}$ & 2016 & Asian & P-B & $\mathrm{HCC}$ & 32 & 55 & 22 & 23 & 66 & 38 \\
\hline & rs187238 & Kim et al..$^{31}$ & 2009 & Asian & $\mathrm{H}-\mathrm{B}$ & $\mathrm{HCC}$ & 37 & 17 & 2 & 434 & 122 & 2 \\
\hline & rs187238 & Teixeira et al.[35] & 2013 & mixed & P-B & $\mathrm{HCC}$ & 57 & 48 & 7 & 100 & 84 & 18 \\
\hline & rs187238 & Lau et al. 27 & 2016 & Asian & $\mathrm{H}-\mathrm{B}$ & $\mathrm{HCC}$ & 266 & 73 & 3 & 476 & 78 & 5 \\
\hline & rs187238 & Bao et al. 25 & 2015 & Asian & P-B & $\mathrm{HCC}$ & 122 & 28 & 3 & 106 & 54 & 5 \\
\hline & rs187238 & Migita et al. ${ }^{29}$ & 2009 & Asian & P-B & $\mathrm{HCC}$ & 43 & 3 & 1 & 52 & 10 & 1 \\
\hline & rs187238 & Chen et al. ${ }^{26}$ & 2012 & Asian & P-B & $\mathrm{HCC}$ & 159 & 59 & 10 & 173 & 115 & 12 \\
\hline & rs187238 & Karra et al. ${ }^{28}$ & 2015 & African & P-B & $\mathrm{HCC}$ & 123 & 134 & 14 & 159 & 108 & 13 \\
\hline & rs187238 & Zhang et al. ${ }^{32}$ & 2016 & Asian & P-B & $\mathrm{HCC}$ & 82 & 25 & 2 & 99 & 24 & 4 \\
\hline \multirow[t]{8}{*}{$I F N-\gamma$} & rs2430561 & Teixeira et al. ${ }^{30}$ & 2013 & Caucasian & P-B & $\mathrm{HCC}$ & 40 & 50 & 21 & 79 & 82 & 41 \\
\hline & rs2430561 & Kim et al..$^{35}$ & 2013 & Asian & H-B & $\mathrm{HCC}$ & 133 & 31 & 6 & 131 & 38 & 2 \\
\hline & rs 2430561 & Migita et al. ${ }^{36}$ & 2005 & Asian & $\mathrm{H}-\mathrm{B}$ & $\mathrm{HCC}$ & 41 & 7 & 0 & 157 & 31 & 0 \\
\hline & rs 2430561 & Ben-Ari et al. ${ }^{39}$ & 2003 & Caucasian & P-B & $\mathrm{HCC}$ & 3 & 7 & 0 & 18 & 24 & 6 \\
\hline & rs2430561 & Nieters et al..$^{37}$ & 2005 & Asian & $\mathrm{H}-\mathrm{B}$ & $\mathrm{HCC}$ & 155 & 94 & & 164 & 86 & \\
\hline & rs2430561 & Saxena et al..$^{38}$ & 2014 & Asian & P-B & $\mathrm{HCC}$ & 15 & 28 & 16 & 52 & 77 & 17 \\
\hline & rs 2430561 & Bouzgarrou et al. ${ }^{34}$ & 2009 & African & P-B & $\mathrm{HCC}$ & 17 & 21 & 20 & 33 & 47 & 23 \\
\hline & rs2430561 & Bahgat et al. ${ }^{33}$ & 2015 & Egyptian & P-B & $\mathrm{HCC}$ & 10 & 24 & 16 & 6 & 15 & 4 \\
\hline \multirow[t]{3}{*}{ IL12A } & rs568408 & Elsayed et al. ${ }^{40}$ & 2016 & Egyptian & P-B & $\mathrm{HCC}$ & 42 & 26 & 10 & 84 & 7 & 1 \\
\hline & rs568408 & Tan et al. ${ }^{42}$ & 2015 & Asian & P-B & $\mathrm{HCC}$ & 313 & 76 & 6 & 511 & 161 & 14 \\
\hline & rs568408 & Liu et al. ${ }^{41}$ & 2011 & Asian & P-B & $\mathrm{HCC}$ & 504 & 277 & 21 & 631 & 220 & 10 \\
\hline \multirow[t]{7}{*}{ IL12B } & rs3212227 & Saxena et al. ${ }^{38}$ & 2014 & Asian & P-B & $\mathrm{HCC}$ & 19 & 31 & 9 & 63 & 71 & 14 \\
\hline & rs3212227 & Elsayed et al. ${ }^{40}$ & 2016 & Egyptian & P-B & $\mathrm{HCC}$ & 41 & 22 & 15 & 38 & 40 & 14 \\
\hline & rs3212227 & Nieters et al..$^{37}$ & 2005 & Asian & H-B & $\mathrm{HCC}$ & 56 & 193 & & 72 & 178 & \\
\hline & rs3212227 & Tan et al. ${ }^{42}$ & 2015 & Asian & P-B & $\mathrm{HCC}$ & 104 & 201 & 90 & 200 & 347 & 139 \\
\hline & rs3212227 & Ognjanovic et al. ${ }^{43}$ & 2009 & mixed & P-B & $\mathrm{HCC}$ & 57 & 60 & & 128 & 95 & \\
\hline & rs3212227 & Liu et al. ${ }^{41}$ & 2011 & Asian & P-B & $\mathrm{HCC}$ & 249 & 422 & 160 & 272 & 414 & 158 \\
\hline & rs3212227 & Yang et al. ${ }^{44}$ & 2011 & Asian & $\mathrm{H}-\mathrm{B}$ & $\mathrm{HCC}$ & 156 & 309 & 143 & 195 & 302 & 115 \\
\hline \multirow[t]{6}{*}{ STAT4 } & rs7574865 & Chanthra et al..$^{45}$ & 2015 & Asian & P-B & $\mathrm{HCC}$ & 19 & 86 & 87 & 28 & 100 & 62 \\
\hline & rs7574865 & Chen et al..$^{21}$ & 2013 & Asian & H-B & $\mathrm{HCC}$ & 35 & 217 & 249 & 75 & 327 & 370 \\
\hline & rs7574865 & Chen et al. ${ }^{46}$ & 2015 & Asian & P-B & $\mathrm{HCC}$ & 40 & 211 & 257 & 343 & 1333 & 1298 \\
\hline & rs7574865 & Clark et al. ${ }^{20}$ & 2013 & Asian & H-B & $\mathrm{HCC}$ & 20 & 102 & 117 & 28 & 92 & 86 \\
\hline & rs7574865 & Kim et al. ${ }^{47}$ & 2015 & Asian & P-B & $\mathrm{HCC}$ & 20 & 103 & 160 & 306 & 1251 & 1293 \\
\hline & rs7574865 & Liao et al. ${ }^{48}$ & 2014 & Asian & P-B & $\mathrm{HCC}$ & 25 & 93 & 104 & 27 & 113 & 97 \\
\hline
\end{tabular}

P-B: population-based; H-B: hospital-based; HCC: Hepatocellular Carcinoma; W: wild allele; M: mutant allele.

Table 2. Results of meta-analysis.

\begin{tabular}{|c|c|c|c|c|c|c|c|c|}
\hline Gene & Polymorphism & Comparison & Subgroup & $\mathbf{N}$ & $P_{\mathrm{H}}$ & $P_{\mathrm{A}}$ & Random & Fixed \\
\hline \multirow[t]{25}{*}{ IL18 } & rs187238 & M vs. W & Overall & 8 & 0.000 & $9.976 \times 10^{-1}$ & $1.000(0.736-1.359)$ & $1.033(0.904-1.181)$ \\
\hline & rs187238 & M vs. W & Asian & 6 & 0.000 & $8.103 \times 10^{-1}$ & $0.948(0.615-1.462)$ & $0.951(0.801-1.130)$ \\
\hline & rs187238 & M vs. W & $\mathrm{H}-\mathrm{B}$ & 2 & 0.577 & $7.535 \times 10^{-4}$ & $1.604(1.223-2.103)$ & $1.597(1.216-2.096)$ \\
\hline & rs187238 & M vs. W & P-B & 6 & 0.002 & $3.018 \times 10^{-1}$ & 0.841 (0.605-1.168) & $0.906(0.777-1.056)$ \\
\hline & rs187238 & M vs. W & $\mathrm{Y}$ & 7 & 0.000 & $6.355 \times 10^{-1}$ & $0.926(0.674-1.272)$ & $0.997(0.868-1.145)$ \\
\hline & rs187238 & WM vs. WW & Overall & 8 & 0.000 & $9.445 \times 10^{-1}$ & $0.986(0.657-1.480)$ & $1.054(0.897-1.240)$ \\
\hline & rs187238 & WM vs. WW & Asian & 6 & 0.000 & $6.487 \times 10^{-1}$ & $0.881(0.509-1.522)$ & $0.924(0.757-1.129)$ \\
\hline & rs187238 & WM vs. WW & $\mathrm{H}-\mathrm{B}$ & 2 & 0.946 & $1.058 \times 10^{-3}$ & $1.665(1.227-2.258)$ & $1.665(1.227-2.259)$ \\
\hline & rs187238 & WM vs. WW & P-B & 6 & 0.000 & $4.054 \times 10^{-1}$ & $0.814(0.501-1.322)$ & $0.887(0.733-1.074)$ \\
\hline & rs 187238 & WM vs. WW & $\mathrm{Y}$ & 7 & 0.000 & $7.038 \times 10^{-1}$ & $0.917(0.588-1.432)$ & $1.023(0.865-1.209)$ \\
\hline & rs187238 & WM+MM vs. WW & Overall & 8 & 0.000 & $9.645 \times 10^{-1}$ & 0.991 (0.671-1.463) & $1.050(0.897-1.228)$ \\
\hline & rs187238 & WM+MM vs. WW & Asian & 6 & 0.000 & $7.008 \times 10^{-1}$ & $0.903(0.535-1.522)$ & $0.933(0.769-1.132)$ \\
\hline & rs187238 & WM+MM vs. WW & H-B & 2 & 0.790 & $6.950 \times 10^{-4}$ & $1.678(1.246-2.260)$ & $1.676(1.243-2.258)$ \\
\hline & rs187238 & $\mathrm{WM}+\mathrm{MM}$ vs. WW & P-B & 6 & 0.000 & $3.724 \times 10^{-1}$ & $0.815(0.519-1.278)$ & $0.883(0.735-1.062)$ \\
\hline & rs187238 & $\mathrm{WM}+\mathrm{MM}$ vs. WW & Y & 7 & 0.000 & $6.719 \times 10^{-1}$ & $0.913(0.601-1.389)$ & $1.011(0.860-1.189)$ \\
\hline & rs187238 & MM vs. WW & Overall & 8 & 0.298 & $9.495 \times 10^{-1}$ & $1.034(0.641-1.667)$ & $0.987(0.654-1.488)$ \\
\hline & rs187238 & MM vs. WW & Asian & 6 & 0.212 & $8.754 \times 10^{-1}$ & $1.086(0.516-2.283)$ & $0.955(0.540-1.691)$ \\
\hline & rs 187238 & MM vs. WW & $\mathrm{H}-\mathrm{B}$ & 2 & 0.051 & $3.368 \times 10^{-1}$ & $3.216(0.296-34.892)$ & $1.934(0.618-6.050)$ \\
\hline & rs 187238 & MM vs. WW & P-B & 6 & 0.806 & $6.383 \times 10^{-1}$ & $0.906\left(0.58^{-1} .416\right)$ & $0.900(0.579-1.398)$ \\
\hline & rs187238 & MM vs. WW & Y & 7 & 0.885 & $6.731 \times 10^{-1}$ & $0.920(0.600-1.408)$ & $0.913(0.599-1.392)$ \\
\hline & rs187238 & MM vs. WM+WW & Overall & 8 & 0.431 & $9.058 \times 10^{-1}$ & $1.012(0.671-1.526)$ & $0.976(0.650-1.464)$ \\
\hline & rs187238 & MM vs. WM+WW & Asian & 6 & 0.299 & $8.563 \times 10^{-1}$ & $1.145(0.587-2.234)$ & $1.054(0.598-1.857)$ \\
\hline & rs187238 & MM vs. WM+WW & H-B & 2 & 0.055 & $3.757 \times 10^{-1}$ & $2.878(0.278-29.858)$ & $1.777(0.570-5.542)$ \\
\hline & rs187238 & MM vs. WM+WW & P-B & 6 & 0.921 & $6.329 \times 10^{-1}$ & $0.906(0.584-1.405)$ & $0.900(0.583-1.389)$ \\
\hline & rs187238 & $M M$ vs. WM+WW & $\mathrm{Y}$ & 7 & 0.963 & $6.419 \times 10^{-1}$ & $0.912(0.600-1.388)$ & $0.906(0.598-1.373)$ \\
\hline
\end{tabular}




\begin{tabular}{|c|c|c|c|c|c|c|c|c|}
\hline Gene & Polymorphism & Comparison & Subgroup & $\mathbf{N}$ & $P_{\mathrm{H}}$ & $P_{\mathrm{A}}$ & Random & Fixed \\
\hline & rs1946518 & W vs. M & Overall & 7 & 0.012 & $2.739 \times 10^{-1}$ & $1.107(0.923-1.329)$ & $1.125(1.014-1.249)$ \\
\hline & rs1946518 & W vs. $M$ & Asian & 5 & 0.083 & $9.618 \times 10^{-1}$ & $0.995(0.822-1.206)$ & $1.024(0.905-1.158)$ \\
\hline & rs1946518 & W vs. $M$ & P-B & 6 & 0.009 & $3.427 \times 10^{-1}$ & $1.117(0.889-1.404)$ & $1.163(1.027-1.317)$ \\
\hline & rs1946518 & W vs. $M$ & $\mathrm{Y}$ & 6 & 0.019 & $5.521 \times 10^{-1}$ & $1.060(0.875-1.285)$ & $1.094(0.980-1.220)$ \\
\hline & rs1946518 & WM vs. WW & Overall & 7 & 0.248 & $7.791 \times 10^{-2}$ & $1.162(0.945-1.430)$ & $1.169(0.983-1.391)$ \\
\hline & rs1946518 & WM vs. WW & Asian & 5 & 0.269 & $5.398 \times 10^{-1}$ & $1.063(0.823-1.373)$ & $1.069(0.864-1.322)$ \\
\hline & rs1946518 & WM vs. WW & P-B & 6 & 0.229 & $4.382 \times 10^{-2}$ & $1.206(0.939-1.549)$ & $1.235(1.006-1.517)$ \\
\hline & rs1946518 & WM vs. WW & Y & 6 & 0.164 & $1.043 \times 10^{-1}$ & $1.152(0.900-1.473)$ & $1.166(0.969-1.404)$ \\
\hline & rs1946518 & WM+MM vs. WW & Overall & 7 & 0.057 & $2.034 \times 10^{-1}$ & $1.176(0.916-1.510)$ & $1.202(1.019-1.417)$ \\
\hline & rs1946518 & WM+MM vs. WW & Asian & 5 & 0.112 & $5.578 \times 10^{-1}$ & $1.035(0.768-1.396)$ & $1.062(0.869-1.297)$ \\
\hline & rs1946518 & WM+MM vs. WW & P-B & 6 & 0.051 & $2.341 \times 10^{-1}$ & $1.203(0.887-1.633)$ & $1.275(1.048-1.550)$ \\
\hline & rs1946518 & WM+MM vs. WW & Y & 6 & 0.038 & $3.901 \times 10^{-1}$ & $1.135(0.850-1.515)$ & $1.176(0.987-1.401)$ \\
\hline & rs1946518 & MM vs. WW & Overall & 7 & 0.003 & $2.675 \times 10^{-1}$ & $1.268(0.833-1.931)$ & $1.269(1.024-1.573)$ \\
\hline & rs1946518 & MM vs. WW & Asian & 5 & 0.075 & $9.398 \times 10^{-1}$ & $0.985(0.663-1.463)$ & $1.043(0.815-1.335)$ \\
\hline & rs1946518 & MM vs. WW & P-B & 6 & 0.002 & $3.262 \times 10^{-1}$ & $1.307(0.766-2.231)$ & 1.369 (1.054-1.777) \\
\hline & rs1946518 & MM vs. WW & Y & 6 & 0.016 & $5.732 \times 10^{-1}$ & $1.123(0.751-1.679)$ & $1.182(0.946-1.477)$ \\
\hline & rs1946518 & MM vs. WM+WW & Overall & 7 & 0.029 & $3.453 \times 10^{-1}$ & 1.155 (0.856-1.559) & $1.141(0.953-1.366)$ \\
\hline & rs1946518 & MM vs. WM+WW & Asian & 5 & 0.350 & $9.856 \times 10^{-1}$ & $0.996(0.799-1.240)$ & $1.002(0.818-1.227)$ \\
\hline & rs1946518 & MM vs. WM+WW & P-B & 6 & 0.017 & $4.088 \times 10^{-1}$ & $1.179(0.797-1.744)$ & $1.178(0.945-1.469)$ \\
\hline & rs1946518 & MM vs. WM+WW & Y & 6 & 0.183 & $4.279 \times 10^{-1}$ & $1.062(0.833-1.353)$ & $1.078(0.895-1.298)$ \\
\hline \multirow[t]{31}{*}{$I F N-\gamma$} & rs2430561 & W vs. M & Overall & 5 & 0.392 & $3.251 \times 10^{-2}$ & $1.239(1.016-1.511)$ & $1.237(1.018-1.503)$ \\
\hline & rs2430561 & W vs. M & Asian & 2 & 0.126 & $6.921 \times 10^{-2}$ & $1.328(0.822-2.145)$ & $1.336(0.977-1.825)$ \\
\hline & rs2430561 & W vs. M & Caucasian & 2 & 0.344 & $4.878 \times 10^{-1}$ & $1.111(0.824-1.499)$ & $1.111(0.825-1.498)$ \\
\hline & rs2430561 & W vs. M & P-B & 4 & 0.338 & $2.146 \times 10^{-2}$ & $1.300(1.030-1.641)$ & $1.288(1.038-1.598)$ \\
\hline & rs2430561 & $\mathrm{W}$ vs. $\mathrm{M}$ & $\mathrm{Y}$ & 4 & 0.489 & $1.284 \times 10^{-2}$ & 1.359 (1.068-1.730) & $1.358(1.067-1.728)$ \\
\hline & rs2430561 & WM vs. WW & Overall & 5 & 0.796 & $9.487 \times 10^{-1}$ & $1.009(0.752-1.355)$ & $1.010(0.753-1.354)$ \\
\hline & rs2430561 & WM vs. WW & Asian & 2 & 0.324 & $7.893 \times 10^{-1}$ & $0.942(0.614-1.445)$ & $0.944(0.617-1.443)$ \\
\hline & rs2430561 & WM vs. WW & Caucasian & 2 & 0.734 & $5.353 \times 10^{-1}$ & $1.162(0.722-1.870)$ & $1.162(0.723-1.869)$ \\
\hline & rs2430561 & WM vs. WW & P-B & 4 & 0.884 & $5.403 \times 10^{-1}$ & $1.116(0.784-1.589)$ & $1.117(0.785-1.589)$ \\
\hline & rs2430561 & WM vs. WW & Y & 4 & 0.799 & $6.838 \times 10^{-1}$ & $0.928(0.648-1.327)$ & $0.929(0.650-1.326)$ \\
\hline & rs2430561 & WM+MM vs. WW & Overall & 8 & 0.943 & $2.454 \times 10^{-1}$ & $1.131(0.916-1.397)$ & $1.133(0.918-1.398)$ \\
\hline & rs2430561 & WM+MM vs. WW & Asian & 4 & 0.544 & $4.033 \times 10^{-1}$ & $1.114(0.859-1.445)$ & $1.116(0.862-1.445)$ \\
\hline & rs2430561 & WM+MM vs. WW & Caucasian & 3 & 0.959 & $4.531 \times 10^{-1}$ & $1.176(0.770-1.797)$ & $1.176(0.770-1.797)$ \\
\hline & rs2430561 & WM+MM vs. WW & $\mathrm{H}-\mathrm{B}$ & 3 & 0.688 & $7.673 \times 10^{-1}$ & $1.044(0.788-1.383)$ & $1.043(0.788-1.381)$ \\
\hline & rs2430561 & WM+MM vs. WW & P-B & 5 & 0.938 & $1.585 \times 10^{-1}$ & $1.255(0.911-1.727)$ & $1.258(0.914-1.730)$ \\
\hline & rs2430561 & WM+MM vs. WW & $\mathrm{Y}$ & 5 & 0.755 & $3.910 \times 10^{-1}$ & $1.148(0.828-1.594)$ & $1.153(0.833-1.596)$ \\
\hline & rs2430561 & WM+MM vs. WW & Overall & 5 & 0.768 & $3.459 \times 10^{-1}$ & $1.138(0.864-1.498)$ & $1.141(0.867-1.500)$ \\
\hline & rs2430561 & WM+MM vs. WW & Asian & 2 & 0.181 & $5.545 \times 10^{-1}$ & $1.162(0.665-2.032)$ & $1.129(0.755-1.687)$ \\
\hline & rs2430561 & $\mathrm{WM}+\mathrm{MM}$ vs. WW & Caucasian & 2 & 0.872 & $5.184 \times 10^{-1}$ & $1.157(0.744-1.802)$ & $1.157(0.743-1.802)$ \\
\hline & rs2430561 & WM+MM vs. WW & P-B & 4 & 0.853 & $1.790 \times 10^{-1}$ & $1.248(0.899-1.731)$ & $1.251(0.902-1.734)$ \\
\hline & rs2430561 & WM+MM vs. WW & Y & 4 & 0.610 & $4.380 \times 10^{-1}$ & $1.137(0.812-1.591)$ & $1.141(0.817-1.593)$ \\
\hline & rs2430561 & MM vs. WW & Overall & 5 & 0.271 & $1.164 \times 10^{-2}$ & $1.777(1.088-2.903)$ & $1.683(1.123-2.523)$ \\
\hline & rs2430561 & MM vs. WW & Asian & 2 & 0.916 & $3.909 \times 10^{-3}$ & $3.188(1.460-6.964)$ & 3.177 (1.449-6.968) \\
\hline & rs2430561 & MM vs. WW & Caucasian & 2 & 0.298 & $6.153 \times 10^{-1}$ & $1.187(0.615-2.293)$ & $1.163(0.645-2.098)$ \\
\hline & rs2430561 & MM vs. WW & P-B & 4 & 0.198 & $2.618 \times 10^{-2}$ & $1.725(0.989-3.011)$ & $1.610(1.058-2.449)$ \\
\hline & rs2430561 & MM vs. WW & Y & 4 & 0.754 & $1.450 \times 10^{-3}$ & $2.375(1.393-4.050)$ & $2.372(1.394-4.035)$ \\
\hline & rs2430561 & MM vs. WM+WW & Overall & 5 & 0.144 & $7.219 \times 10^{-3}$ & $1.785(1.075-2.966)$ & $1.624(1.140-2.313)$ \\
\hline & rs 2430561 & MM vs. WM+WW & Asian & 2 & 0.920 & $2.773 \times 10^{-3}$ & $2.871(1.438-5.731)$ & $2.880(1.440-5.760)$ \\
\hline & rs2430561 & MM vs. WM+WW & Caucasian & 2 & 0.151 & $6.576 \times 10^{-1}$ & $1.294(0.512-3.269)$ & $1.123(0.671-1.880)$ \\
\hline & rs2430561 & MM vs. WM+WW & P-B & 4 & 0.102 & $1.646 \times 10^{-2}$ & $1.713(0.979-2.997)$ & $1.562(1.085-2.248)$ \\
\hline & rs2430561 & MM vs. WM+WW & Y & 4 & 0.847 & $2.991 \times 10^{-4}$ & $2.331(1.471-3.691)$ & $2.333(1.474-3.693)$ \\
\hline \multirow[t]{15}{*}{ STAT4 } & rs7574865 & W vs. $\mathrm{M}$ & Overall & 6 & 0.395 & $1.760 \times 10^{-8}$ & $1.270(1.166-1.384)$ & $1.272(1.170-1.382)$ \\
\hline & rs7574865 & W vs. M & H-B & 2 & 0.300 & $3.909 \times 10^{-2}$ & $1.172(1.003-1.370)$ & 1.169 (1.008-1.356) \\
\hline & rs7574865 & W vs. $M$ & P-B & 4 & 0.503 & $7.210 \times 10^{-8}$ & $1.320(1.193-1.461)$ & $1.321(1.194-1.462)$ \\
\hline & rs7574865 & WM vs. WW & Overall & 6 & 0.845 & $9.838 \times 10^{-3}$ & $1.302(1.064-1.592)$ & $1.303(1.066-1.593)$ \\
\hline & rs7574865 & WM vs. WW & H-B & 2 & 0.824 & $3.922 \times 10^{-2}$ & $1.462(1.020-2.097)$ & $1.461(1.019-2.096)$ \\
\hline & rs7574865 & WM vs. WW & P-B & 4 & 0.706 & $8.236 \times 10^{-2}$ & $1.234(0.968-1.574)$ & $1.239(0.973-1.577)$ \\
\hline & rs7574865 & WM+MM vs. WW & Overall & 6 & 0.841 & $7.092 \times 10^{-5}$ & $1.470(1.213-1.781)$ & $1.474(1.217-1.785)$ \\
\hline & rs7574865 & WM+MM vs. WW & $\mathrm{H}-\mathrm{B}$ & 2 & 0.624 & $1.768 \times 10^{-2}$ & $1.520(1.077-2.145)$ & $1.518(1.075-2.143)$ \\
\hline & rs7574865 & WM+MM vs. WW & P-B & 4 & 0.623 & $1.354 \times 10^{-3}$ & 1.447 (1.148-1.824) & $1.456(1.157-1.833)$ \\
\hline & rs7574865 & MM vs. WW & Overall & 6 & 0.755 & $6.561 \times 10^{-7}$ & $1.651(1.352-2.016)$ & $1.658(1.359-2.024)$ \\
\hline & rs7574865 & MM vs. WW & H-B & 2 & 0.479 & $1.355 \times 10^{-2}$ & $1.574(1.101-2.251)$ & $1.571(1.098-2.248)$ \\
\hline & rs7574865 & MM vs. WW & P-B & 4 & 0.564 & $1.593 \times 10^{-5}$ & $1.687(1.326-2.146)$ & $1.696(1.334-2.156)$ \\
\hline & rs7574865 & $M M$ vs. WM+WW & Overall & 6 & 0.249 & $4.680 \times 10^{-7}$ & $1.330(1.168-1.516)$ & $1.322(1.186-1.473)$ \\
\hline & rs7574865 & MM vs. WM+WW & H-B & 2 & 0.324 & $1.889 \times 10^{-1}$ & $1.138(0.938-1.380)$ & $1.138(0.938-1.380)$ \\
\hline & rs7574865 & MM vs. WM+WW & P-B & 4 & 0.518 & $1.961 \times 10^{-7}$ & $1.417(1.242-1.616)$ & 1.417 (1.243-1.617) \\
\hline \multirow[t]{7}{*}{ IL12A } & rs568408 & W vs. M & Overall & 3 & 0.000 & $1.096 \times 10^{-1}$ & $1.926(0.863-4.299)$ & $1.363(1.180-1.574)$ \\
\hline & rs568408 & W vs. $M$ & Asian & 2 & 0.000 & $7.654 \times 10^{-1}$ & $1.105(0.573-2.130)$ & $1.235(1.064-1.434)$ \\
\hline & rs568408 & WM vs. WW & Overall & 3 & 0.000 & $1.450 \times 10^{-1}$ & $1.804(0.816-3.988)$ & $1.342(1.135-1.587)$ \\
\hline & rs568408 & WM vs. WW & Asian & 2 & 0.000 & $7.665 \times 10^{-1}$ & $1.112(0.552-2.242)$ & $1.245(1.048-1.479)$ \\
\hline & rs568408 & WM+MM vs. WW & Overall & 3 & 0.000 & $1.193 \times 10^{-1}$ & $1.982(0.838-4.689)$ & $1.386(1.178-1.631)$ \\
\hline & rs568408 & WM+MM vs. WW & Asian & 2 & 0.000 & $7.580 \times 10^{-1}$ & $1.123(0.538-2.345)$ & $1.261(1.066-1.492)$ \\
\hline & rs568408 & WM+MM vs. WW & Overall & 3 & 0.000 & $1.193 \times 10^{-1}$ & $1.982(0.838-4.689)$ & $1.386(1.178-1.631)$ \\
\hline
\end{tabular}




\begin{tabular}{|c|c|c|c|c|c|c|c|c|}
\hline Gene & Polymorphism & Comparison & Subgroup & $\mathbf{N}$ & $P_{\mathrm{H}}$ & $P_{\mathrm{A}}$ & Random & Fixed \\
\hline \multirow{24}{*}{ IL12B } & rs568408 & WM+MM vs. WW & Asian & 2 & 0.000 & $7.580 \times 10^{-1}$ & $1.123(0.538-2.345)$ & $1.261(1.066-1.492)$ \\
\hline & rs568408 & MM vs. WW & Overall & 3 & 0.007 & $2.029 \times 10^{-1}$ & $2.574(0.601-11.033)$ & $2.151(1.285-3.603)$ \\
\hline & rs568408 & MM vs. WW & Asian & 2 & 0.035 & $6.075 \times 10^{-1}$ & $1.404(0.384-5.130)$ & $1.576(0.897-2.771)$ \\
\hline & rs568408 & MM vs. WM+WW & Overall & 3 & 0.025 & $2.298 \times 10^{-1}$ & $2.158(0.615-7.568)$ & $1.957(1.160-3.300)$ \\
\hline & rs568408 & MM vs. WM+WW & Asian & 2 & 0.072 & $5.886 \times 10^{-1}$ & $1.356(0.450-4.085)$ & $1.487(0.843-2.622)$ \\
\hline & rs3212227 & W vs. M & Overall & 5 & 0.301 & $6.458 \times 10^{-3}$ & $1.129(1.021-1.247)$ & $1.127(1.034-1.228)$ \\
\hline & rs3212227 & W vs. M & Asian & 4 & 0.344 & $3.593 \times 10^{-3}$ & 1.141 (1.039-1.254) & $1.139(1.043-1.243)$ \\
\hline & rs3212227 & W vs. M & P-B & 4 & 0.439 & $1.334 \times 10^{-1}$ & $1.081(0.977-1.197)$ & $1.081(0.976-1.197)$ \\
\hline & rs3212227 & WM vs. WW & Overall & 5 & 0.156 & $7.995 \times 10^{-2}$ & $1.115(0.914-1.360)$ & $1.133(0.985-1.302)$ \\
\hline & rs3212227 & WM vs. WW & Asian & 4 & 0.769 & $2.749 \times 10^{-2}$ & $1.174(1.018-1.354)$ & $1.174(1.018-1.354)$ \\
\hline & rs3212227 & WM vs. WW & P-B & 4 & 0.138 & $3.564 \times 10^{-1}$ & $1.048(0.805-1.365)$ & $1.080(0.917-1.273)$ \\
\hline & rs3212227 & WM+MM vs. WW & Overall & 7 & 0.282 & $2.390 \times 10^{-3}$ & $1.210(1.049-1.394)$ & $1.205(1.068-1.360)$ \\
\hline & rs3212227 & WM+MM vs. WW & Asian & 5 & 0.624 & $1.947 \times 10^{-3}$ & $1.224(1.077-1.391)$ & $1.224(1.077-1.391)$ \\
\hline & rs3212227 & WM+MM vs. WW & $\mathrm{H}-\mathrm{B}$ & 2 & 0.906 & $3.978 \times 10^{-3}$ & $1.366(1.105-1.688)$ & $1.366(1.105-1.688)$ \\
\hline & rs3212227 & WM+MM vs. WW & P-B & 5 & 0.243 & $8.896 \times 10^{-2}$ & $1.139(0.941-1.378)$ & $1.136(0.981-1.315)$ \\
\hline & rs3212227 & WM+MM vs. WW & Overall & 5 & 0.181 & $1.900 \times 10^{-2}$ & $1.162(0.970-1.392)$ & $1.170(1.026-1.335)$ \\
\hline & rs3212227 & WM+MM vs. WW & Asian & 4 & 0.537 & $6.362 \times 10^{-3}$ & $1.206(1.054-1.381)$ & $1.207(1.054-1.381)$ \\
\hline & rs3212227 & WM+MM vs. WW & P-B & 4 & 0.221 & $2.023 \times 10^{-1}$ & $1.096(0.884-1.358)$ & $1.106(0.947-1.291)$ \\
\hline & rs3212227 & MM vs. WW & Overall & 5 & 0.429 & $6.126 \times 10^{-3}$ & $1.278(1.073-1.522)$ & $1.277(1.072-1.520)$ \\
\hline & rs3212227 & MM vs. WW & Asian & 4 & 0.323 & $5.045 \times 10^{-3}$ & $1.301(1.067-1.586)$ & $1.291(1.080-1.543)$ \\
\hline & rs3212227 & MM vs. WW & P-B & 4 & 0.605 & $1.250 \times 10^{-1}$ & $1.179(0.957-1.451)$ & $1.177(0.956-1.448)$ \\
\hline & rs3212227 & MM vs. WM+WW & Overall & 5 & 0.629 & $3.630 \times 10^{-2}$ & $1.176(1.011-1.367)$ & $1.175(1.010-1.367)$ \\
\hline & rs3212227 & MM vs. WM+WW & Asian & 4 & 0.476 & $4.563 \times 10^{-2}$ & $1.170(1.004-1.365)$ & $1.170(1.003-1.364)$ \\
\hline & rs3212227 & MM vs. WM+WW & P-B & 4 & 0.681 & $2.405 \times 10^{-1}$ & $1.116(0.931-1.336)$ & $1.114(0.930-1.335)$ \\
\hline
\end{tabular}

P-B: population-based; H-B: hospital-based; W: wild allele; M: mutant allele; HWE: Hardy-Weinberg equilibrium (Y: conform to HWE; N: not conform to HWE). Characters with bold mean statistically significant.

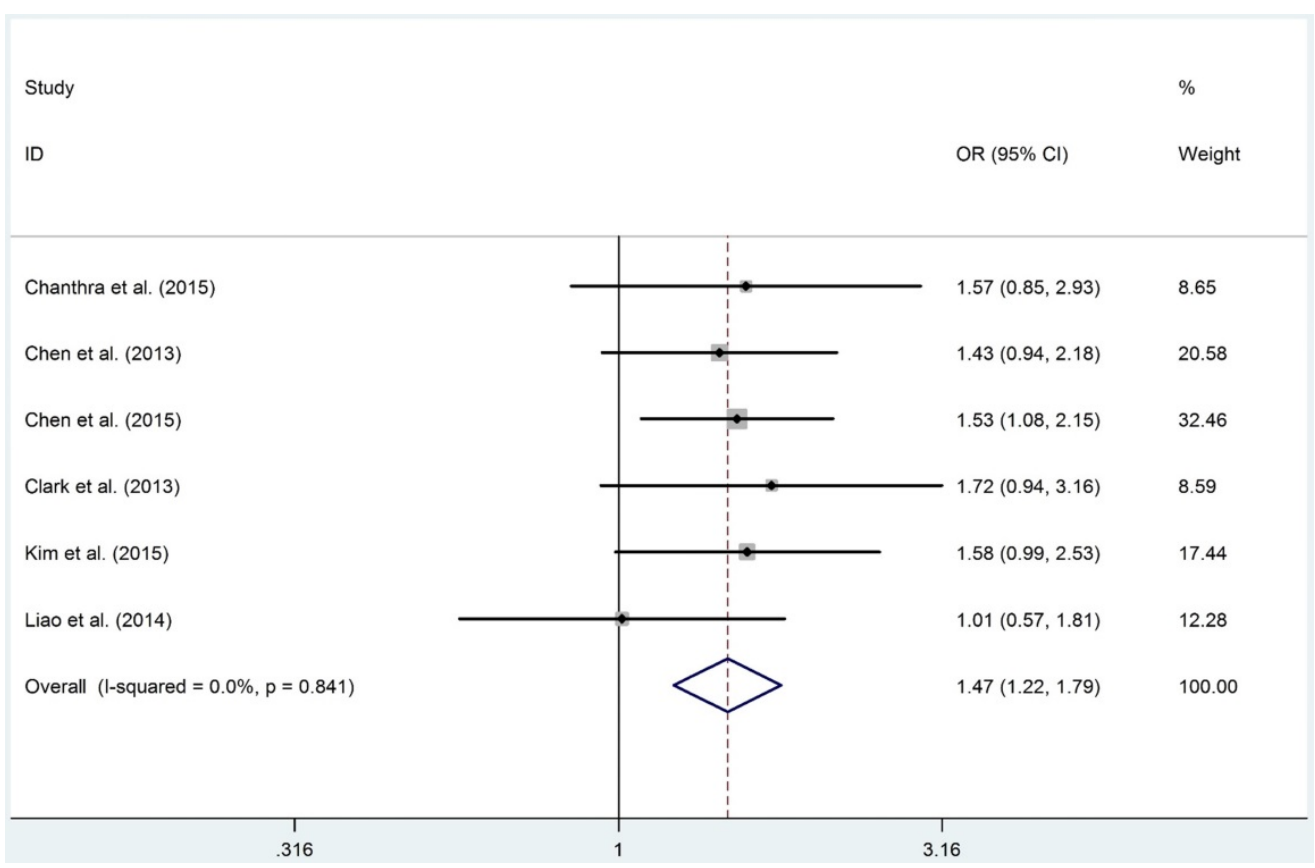

Figure 2. Forest plot of STAT4 gene rs7574865 polymorphism in dominant model (MW+MM vs. WW).

\section{Discussion}

IL-12 coordinates innate and adaptive immune responses in human beings and is regarded as an important immunomodulatory cytokine in immune system. STAT4 promotes the differentiation of naive $\mathrm{CD}^{+}{ }^{+} \mathrm{T}$ cells into Th- 1 and cytotoxicity of NK cells, as well as the T cell proliferation [54], and its activation is mainly triggered by IL-12 signaling. In addition, IL-12 and IL-12R complex could functionally promote the phosphorylation of Jak kinase, promoting cell growth [55]. These genes are key genes of IL12 signaling pathway and could functionally work together to exert their function.

It was worth noting that $\mathrm{HBV}$ carriers have a greater than 100 -fold increased relative risk of developing the HCC [56], and evidence has pointed out that IL-12 signaling pathway plays a pivotal role during anti-HBV-infection, and even on HCC tumorigenesis. In addition, evidence also suggested that genetic variations in genes of IL-12 signaling pathway were associated with HCC risk. Nevertheless, till now, no consistent conclusions had been acquired. Therefore, we collected all the 
available studies and conducted current updated meta-analysis to comprehensively validate the associations between genetic polymorphisms in genes of IL-12 signaling pathway and HCC risk, trying to identify more genetic markers for the screening of HCC.

Here, we identified that STAT4-rs7574865 polymorphism conferred a statistically increased risk of HCC. As for IFN- $\gamma$-rs2430561 polymorphism, although overall analysis failed to identify any positive result, we found that for these studies whose control groups conformed to HWE, were significantly associated with an increased risk of HCC. Besides, subgroup analyses based on source of control also identified that the P-B groups were more susceptible to develop HCC in allelic, homozygous, recessive and dominant models for STAT4-rs7574865 polymorphism, while H-B groups were more susceptible to HCC risk for IL18-rs187238 polymorphism in allelic, heterozygous and dominant models, respectively, suggesting that the source of control was also one of the bias influencer.

STAT4 is the key member of STAT protein family, which could transduce signals of cytokine-receptor complexes, and could regulate the transcription of several genes. Through JAK/STAT signaling pathway, IL-12, IL-23 and IFN-1 could induce the response of STAT4, furthermore, the transcription and expression of a variety of genes would be regulated [57-60]. Currently, the influence of STAT4-rs7574865 polymorphism on HCC tumorigenesis have been performed on several previous studies, but the results were contraverial[25, 50, 51]. The current analysis revealed that the " $\mathrm{M}$ " allele of STAT4-rs7574865 polymorphism conferred to an increased risk of HCC. In addition, the pooled results also demonstrated that MM mutant genotype was 1.651 and 1.330-fold increased risk of HCC than WW and MW+WW genotypes, respectively.

IFN-ץ plays a critical role in liver function, and it could impact the apoptosis and regeneration of hepatocyte [61]. The balance of STAT4 depended IFN- $\gamma$ expression could affect both the antiviral and antitumor processes [62]. In previous study, some incompatible correlations were found between variants in IFN- $\gamma$ gene and the risk of HCC. Saxena et al. [43] reported that the wild genotype (TT) distribution of IFN- $\gamma$-rs2430561 polymorphism had the highest frequency for HCC group (27.12\%), and was significantly higher than controls. On the contrast, no statistically significant difference in $I F N-\gamma$-rs2430561 genotype frequency was presented between chronic hepatitis patients and cirrhotic/HCC group in the study conducted by Bahgat et al.[38]. In current work, the overall pooled results suggested that there was no statistical connection between rs2430561 polymorphism and HCC risk, while the further subgroup analysis by HWE status found that for these studies whose controls conformed to HWE were significantly associated with an increased risk of HCC, suggesting that HWE status influenced the overall results, causing potential bias.

In this meta-analysis, publication retrieval was carefully done according to the pre-set strict inclusion standards. The advantages of current work should not be buried. Firstly, this is the first study concerned the relationships between all the available genetic polymorphisms in genes of IL-12 signaling pathway and HCC risk. Secondly, we used NOS form to evaluate the quality of each registered study, and low quality studies will be eliminated to further raise the credibility of pooled results. Thirdly, stratification analyses were performed based on ethnicity, source of controls and ethnicity, to decrease the impact of heterogeneity sources, thus we could obtain more accurate results. Fourthly, recognized formula was used to adjust the results, avoiding false positive results. Fifthly, sensitivity analysis was conducted to confirm the stability of current conclusions, and Egger's test and Begg's funnel plot were carried out to detect potential publication bias. On the contrast, several disadvantages should also be listed here. In the first place, there were no sufficient samples for several genetic polymorphisms, which might provide an untrustworthy result. What's more, we only enrolled publications written in English or Chinese, missing publications from other languages may cause potential bias. Last but not the least, we failed to obtain the detail histological subtypes of HCC patients, therefore, stratification analysis based on histological type cannot be conducted.

To conclude, the present meta-analysis suggests that the STAT4-rs7574865 polymorphism is a risk factor for HCC patients.

\section{Supplementary Material}

Supplementary figures and tables. http://www.jcancer.org/v09p3583s1.pdf

\section{Acknowledgments}

Study design: L.G., Y.X.; Performed the study: Y.X. and G.L.; Analyzed the data: Y.X. and G.L.; Wrote and revised the paper: Y.X., G.L. and L.G.

\section{Competing Interests}

The authors have declared that no competing interest exists. 


\section{References}

1. Torre LA, Bray F, Siegel RL, Ferlay J, Lortet-Tieulent J, Jemal A. Global Cancer Statistic, 2012. Ca A Cancer Journal for Clinicians. 2015; 65: 87-108.

2. Ma J, Jiang Y, Chen X, Gong G. Langerhans cell histiocytosis misdiagnosed as liver cancer and pituitary tumor in an adult: A case report and brief review of the literature. Oncology letters. 2014; 7: 1602-4.

3. Ferlay J, Soerjomataram I, Dikshit R, Eser S, Mathers C, Rebelo M, et al. Cancer incidence and mortality worldwide: sources, methods and major patterns in GLOBOCAN 2012. International Journal of Cancer. 2015; 136: E359-E86.

4. Qiu XX, Hong Y, Yang DR, Xia M, Zhu HZ, Li QL, et al. Our study was supported by the Changsha City Third Science and Technology Project (K1403380-31, to Chaohui Zuo). ISG15 as a novel prognostic biomarker for hepatitis B virus-related hepatocellular carcinoma. International Journal of Clinical and Experimental Medicine. 2015; 8: 17140-50.

5. Fu S, Wang J, Hu X, Zhou RR, Fu Y, Tang D, et al. Crosstalk between hepatitis $\mathrm{B}$ virus $\mathrm{x}$ and high-mobility group box 1 facilitates autophagy in hepatocytes. Molecular Oncology. 2018.

6. Chen W, Zheng R, Baade PD, Zhang S, Zeng H, Bray F, et al. Cancer statistics in China, 2015. Ca A Cancer Journal for Clinicians. 2016; 66: 115.

7. Luo AJ, Wang FZ, Luo D, Hu DH, Mao P, Xie WZ, et al. Consumption of vegetables may reduce the risk of liver cancer: results from a meta-analysis of case-control and cohort studies. Clinics and research in hepatology and gastroenterology. 2015; 39: 45-51.

8. Zhou Y, Zhou N, Fang W, Huo J. Overexpressed HDGF as an independent prognostic factor is involved in poor prognosis in Chinese patients with liver cancer. Diagnostic pathology. 2010; 5: 58.

9. Huang RX, Duan YY, Hu JA. Fish intake and risk of liver cancer: a meta-analysis. PloS one. 2015; 10: e0096102.

10. He G, Dhar D, Nakagawa H, Font-Burgada J, Ogata H, Jiang Y, et al. Identification of Liver Cancer Progenitors Whose Malignant Progression Depends on Autocrine IL-6 Signaling. Cell. 2013; 155: 384-96.

11. Li S, Sun R, Chen Y, Wei H, Tian Z. TLR2 limits development of hepatocellular carcinoma by reducing IL18-mediated immunosuppression. Cancer Research. 2015; 75: 986.

12. Wang $\mathrm{Q}$, Cheng F, Ma TT, Xiong HY, Li ZW, Xie CL, et al. Interleukin-12 inhibits the hepatocellular carcinoma growth by inducing macrophage polarization to the M1-like phenotype through downregulation of Stat-3. Molecular \& Cellular Biochemistry. 2016; 415: 157.

13. Chehimi I, Trinchieri G. Interleukin-12: A bridge between innate resistance and adaptive immunity with a role in infection and acquired immunodeficiency. Journal of Clinical Immunology. 1994; 14: 149-61.

14. Zhang H, Nie W, Huang F. The Correlation Relationship between P14ARF Gene DNA Methylation and Primary Liver Cancer. Medical science monitor : international medical journal of experimental and clinical research. 2015; 21: $3077-82$.

15. Bacon CM, Petricoin EF, 3rd, Ortaldo JR, Rees RC, Larner AC, Johnston JA, et al. Interleukin 12 induces tyrosine phosphorylation and activation of STAT4 in human lymphocytes. Proc Natl Acad Sci U S A. 1995; 92: 7307-11.

16. Ma X, Chow JM, Gri G, Carra G, Gerosa F, Wolf SF, et al. The interleukin 12 p40 gene promoter is primed by interferon gamma in monocytic cells. J Exp Med. 1996; 183: 147-57.

17. Smeltz RB, Chen J, Huli J, Shevach EM. Regulation of Interleukin (II)-18 Receptor a Chain Expression on Cd4+ T Cells during T Helper (Th)1/Th2 Differentiation Critical Downregulatory Role of IL-4. Journal of Experimental Medicine. 2001; 194: 143-53.

18. Kohka H, Yoshino T, Iwagaki H, Sakuma I, Tanimoto T, Matsuo $\mathrm{Y}$, et al. Interleukin-18/interferon-gamma-inducing factor, a novel cytokine, up-regulates ICAM-1 (CD54) expression in KG-1 cells. J Leukoc Biol. 1998; 64: 519-27.

19. Nakanishi $\mathrm{K}$, Yoshimoto $\mathrm{T}$, Tsutsui $\mathrm{H}$, Okamura H. Interleukin-18 regulates both Th1 and Th2 responses. Annu Rev Immunol. 2001; 19: 423-74.

20. Wang HW, Gao HL, Wei XX, Wang XH. Up-regulation of IL-12 expression in patients with chronic hepatitis B is mediated by the PI3K/Akt pathway. Mol Cell Biochem. 2015; 407: 135-42.

21. Yin D, Wang Y, Sai W, Zhang L, Miao Y, Cao L, et al. HBx-induced miR-21 suppresses cell apoptosis in hepatocellular carcinoma by targeting interleukin-12. Oncol Rep. 2016; 36: 2305-12.

22. Yang $Y, Q i u X Q, Y u$ HP. Polymorphism of TGF- $\beta 1$ and IL-12B gene and risk of hepatocellular cancer in Guangxi: a case-control study. Chinese Journal of Public Health. 2011.

23. Elsayed HM, Nabiel Y, Sheta T. IL12 Gene Polymorphism in Association with Hepatocellular Carcinoma in HCV-infected Egyptian Patients. Immunol Invest. 2017; 46: 123-33.

24. Chanthra N, Payungporn S, Chuaypen N, Piratanantatavorn K, Pinjaroen N, Poovorawan Y, et al. Single Nucleotide Polymorphisms in STAT3 and STAT4 and Risk of Hepatocellular Carcinoma in Thai Patients with Chronic Hepatitis B. Asian Pac J Cancer Prev. 2015; 16: 8405-10.

25. Clark A, Gerlach F, Tong H, Hoan NX, Song LH, Toan NL, et al. A trivial role of STAT4 variant in chronic hepatitis B induced hepatocellular carcinoma. Infection Genetics \& Evolution Journal of Molecular Epidemiology \& Evolutionary Genetics in Infectious Diseases. 2013; 18: 257-61.

26. Chen $K$, Shi W, Xin Z, Wang H, Zhu X, Wu X, et al. Replication of genome wide association studies on hepatocellular carcinoma susceptibility loci in a Chinese population. PloS one. 2013; 8: e77315.
27. Fenaroli G, Garibaldi U, Penco MA. Giochi, scommesse sulla vita, tabelle di mortalita, nascita del calcolo probabilistico, statistica e teoria delle popolazioni. Archive for history of exact sciences. 1981; 25: 329-41.

28. Lau J, Ioannidis JP, Schmid CH. Quantitative synthesis in systematic reviews. Ann Intern Med. 1997; 127: 820-6.

29. Harbord RM, Egger M, Sterne JAC. A modified test for small-study effects in meta-analyses of controlled trials with binary endpoints. Statistics in Medicine. 2006; 25: 3443-57.

30. Bao J, Yu L, Deng Y, Rong C, Liu Y, Huang X, et al. Association between IL - 18 polymorphisms, serum levels, and HBV-related hepatocellular carcinoma in a Chinese population: a retrospective case-control study. Cancer Cell International. 2015; 15: 72.

31. Chen Q, Liao W, Songqing HE, Yuan S, Qin L, Yaqun YU. The correlation between single nucleotide polymorphism of interleukin-18 gene promoter and genetic susceptibility to hepatocellular carcinoma. Immunological Journal. 2012.

32. Hon-Kit L, Ming-Ju H, Yang SF, Wang HL, Wu-Hsien K, Hsiang-Lin L, et al. Association between Interleukin-18 Polymorphisms and Hepatocellular Carcinoma Occurrence and Clinical Progression. International Journal of Medical Sciences. 2016; 13: 556-61.

33. Karra VK, Gumma PK, Chowdhury SJ, Ruttala R, Polipalli SK, Chakravarti A, et al. IL-18 polymorphisms in hepatitis B virus related liver disease. Cytokine. 2015; 73: 277-82.

34. Migita K, Sawakami-Kobayashi K, Maeda Y, Nakao K, Kondoh S, Sugiura M, et al. Interleukin-18 promoter polymorphisms and the disease progression of Hepatitis B virus-related liver disease. Translational Research. 2009; 153: 91-6.

35. Teixeira AC, Jr MC, Marano LA, Deghaide NH, Secaf M, Jr EJ, et al. Alleles and genotypes of polymorphisms of IL-18, TNF- $a$ and IFN- $\gamma$ are associated with a higher risk and severity of hepatocellular carcinoma (HCC) in Brazil. Human Immunology. 2013; 74: 1024-9.

36. Yong SK, Cheong JY, Cho SW, Lee KM, Hwang JC, Oh B, et al. A Functional SNP of the Interleukin-18 Gene Is Associated with the Presence of Hepatocellular Carcinoma in Hepatitis B Virus-Infected Patients. Digestive Diseases \& Sciences. 2009; 54: 2722-8.

37. Zhang QX, Yao YQ, Li SL, Long Q. [Association between interleukin-18 gene polymorphisms and hepatocellular carcinoma caused by hepatitis B virus]. Zhonghua gan zang bing za $\mathrm{zhi}=$ Zhonghua ganzangbing zazhi $=$ Chinese journal of hepatology. 2016; $24: 352$

38. Bahgat NA, Kamal MM, Abdelaziz AO, Mohye MA, Shousha HI, Ahmed MM, et al. Interferon- $\gamma$ and Interleukin-10 Gene Polymorphisms are not Predictors of Chronic Hepatitis C (Genotype-4) Disease Progression. Asian Pacific Journal of Cancer Prevention Apjcp. 2015; 16: 5025.

39. Bouzgarrou N, Hassen E, Farhat K, Bahri O, Gabbouj S, Maamouri N, et al. Combined analysis of interferon- $\gamma$ and interleukin- 10 gene polymorphisms and chronic hepatitis C severity. Human Immunology. 2009; 70: 230-6.

40. Kim HJ, Chung JH, Shin HP, Jeon JW, Park JJ, Cha JM, et al. Polymorphisms of interferon gamma gene and risk of hepatocellular carcinoma in Korean patients with chronic hepatitis B viral infection. Hepatogastroenterology. 2013; 60: 1117-20.

41. Migita K, Miyazoe S, Maeda Y, Daikoku M, Abiru S, Ueki T, et al. Cytokine gene polymorphisms in Japanese patients with hepatitis $\mathrm{B}$ virus infection--association between TGF-beta1 polymorphisms and hepatocellular carcinoma. Journal of Hepatology. 2005; 42: 505-10.

42. Nieters A, Yuan JM, Sun CL, Zhang ZQ, Stoehlmacher J, Govindarajan S, et al. Effect of cytokine genotypes on the hepatitis B virus-hepatocellular carcinoma association. Cancer. 2010; 103: 740-8.

43. Saxena R, Chawla YK, Verma I, Kaur J. IFN-Y (+874) and not TNF-a (-308) is associated with HBV-HCC risk in India. Molecular \& Cellular Biochemistry. 2014:385: 297-307.

44. Z B, Mor E, Papo O, Kfir B, Sulkes J, Tambur AR, et al. Cytokine gene polymorphisms in patients infected with hepatitis B virus. American Journal of Gastroenterology. 2003; 98: 144-50,

45. Elsayed HM, Nabiel Y, Sheta T. IL12 Gene Polymorphism in Association with Hepatocellular Carcinoma in HCV-infected Egyptian Patients. Immunological Investigations. 2016; 46: 123-33

46. Liu L, Xu Y, Liu Z, Chen J, Zhang Y, Zhu J, et al. IL12 polymorphisms, HBV infection and risk of hepatocellular carcinoma in a high-risk Chinese population. International Journal of Cancer. 2013; 48: 125-31.

47. Tan A, Gao Y, Yao Z, Su S, Jiang Y, Xie Y, et al. Genetic variants in IL12 influence both hepatitis B virus clearance and HBV-related hepatocellular carcinoma development in a Chinese male population. Tumour Biology the Journal of the International Society for Oncodevelopmental Biology \& Medicine. 2016; 37: 6343.

48. Ognjanovic S, Yuan JM, Chaptman AK, Fan Y, Yu MC. Genetic polymorphisms in the cytokine genes and risk of hepatocellular carcinoma in low-risk non-Asians of USA. Carcinogenesis. 2009; 30: 758.

49. Yan $Y$, Qiu XQ, Hong-Ping YU. Polymorphism of TGF- $\beta 1$ and IL-12B gene and risk of hepatocellular cancer in Guangxi--a case-control study. Chinese Journal of Public Health. 2011.

50. Chanthra N, Payungporn S, Chuaypen N, Piratanantatavorn K, Pinjaroen N, Poovorawan Y, et al. Single Nucleotide Polymorphisms in STAT3 and STAT4 and Risk of Hepatocellular Carcinoma in Thai Patients with Chronic Hepatitis B. Asian Pacific Journal of Cancer Prevention Apjcp. 2015; 16: 8405. 
51. Chen W, Wang M, Zhang Z, Tang H, Zuo X, Meng X, et al. Replication the association of 2q32.2-q32.3 and 14q32.11 with hepatocellular carcinoma. Gene. 2015; 561: 63-7.

52. Kim LH, Cheong HS, Namgoong S, Kim JO, Kim JH, Park BL, et al. Replication of genome wide association studies on hepatocellular carcinoma susceptibility loci of STAT4 and HLA-DQ in a Korean population. Infection Genetics \& Evolution Journal of Molecular Epidemiology \& Evolutionary Genetics in Infectious Diseases. 2015; 33: 72-6.

53. Liao Y, Cai B, Li Y, Chen J, Tao C, Huang H, et al. Association of HLA-DP/DQ and STAT4 polymorphisms with HBV infection outcomes and a mini meta-analysis. Plos One. 2014; 9: e111677.

54. Watford WT, Hissong BD, Bream JH, Kanno Y, Muul L, O'Shea JJ. Signaling by IL-12 and IL-23 and the immunoregulatory roles of STAT4. Immunol Rev. 2004; 202: 139-56.

55. Hamza T, Barnett JB, Li B. Interleukin 12 a key immunoregulatory cytokine in infection applications. Int J Mol Sci. 2010; 11: 789-806.

56. El-Serag HB. Epidemiology of viral hepatitis and hepatocellular carcinoma. Gastroenterology. 2012; 142: 1264-73 e1.

57. O'Shea JJ, Holland SM, Staudt LM. JAKs and STATs in immunity, immunodeficiency, and cancer. N Engl J Med. 2013; 368: 161-70.

58. Zhou RR, Kuang XY, Huang Y, Li N, Zou MX, Tang DL, et al. Potential role of High mobility group box 1 in hepatocellular carcinoma. Cell Adh Migr. 2014; 8: 493-8.

59. You Y, Fan X, Huang $Z, L i \quad N$. Effect of CpG motif containing oligodeoxynucleotides on dendritic cell function in patients with chronic hepatitis B. Zhong Nan Da Xue Xue Bao Yi Xue Ban. 2009; 34: 461-7.

60. Chen L, Li CZ, Peng ZQ, Zhao JX, Gong GZ, Tan DM. miR-197 Expression in Peripheral Blood Mononuclear Cells from Hepatitis B Virus-Infected Patients. Gut and Liver. 2013; 7: 335-42.

61. Horras CJ, Lamb CL, Mitchell KA. Regulation of hepatocyte fate by interferon-gamma. Cytokine Growth Factor Rev. 2011; 22: 35-43.

62. Saha B, Jyothi Prasanna S, Chandrasekar B, Nandi D. Gene modulation and immunoregulatory roles of interferon gamma. Cytokine. 2010; 50: 1-14 